R. J. Cohen and W. T. Sullivan, III, eds.

\title{
Light Pollution: Changing the Situation to Everyone's Advantage
}

\author{
David L. Crawford \\ International Dark-Sky Association, 3225 North First Avenue, Tuscon, \\ AZ 85719-2103, USA
}

\begin{abstract}
There is no question of the need for outdoor lighting to improve the effectiveness of our night-time environment. However, too much of the lighting installed to try to meet this need actually compromises the purpose, and it too often adversely affects the night-time environment, including our view of the stars and of the Universe above us. This urban sky glow severe impacts on all of astronomy, amateur and professional, as well as those of the public who enjoy and profit by the beauty offered by a prime dark sky. In the present paper, I review the issues involved and suggest guidelines to minimize these negative aspects of poor night-time lighting. With good outdoor lighting we all win.
\end{abstract}

\section{Introduction}

Outdoor lighting is an essential component of our night-time environment. There are few places in the world today where it is not widely used. The reasons are rather obvious, but they all relate to improved visibility at night. While the eye is a marvelous instrument, and very sensitive over a wide range of lighting levels, we often need to supplement the existing light so as to improve visibility. (It is remarkable, though, how well we can see even by moonlight or less if there are no sources of glare to compromise our vision and the adaptation level of the eye.) The improved visibility we are seeking is for the purpose of moving around safely at night, walking or driving. We can see the ground better, as well as any obstacles such as curbs, unexpected holes, stones, or other objects. In addition, we feel safer, especially in our cities where today the fear of crime can be high. By being able to see better, we expect to be safer, and sometimes we are.

For these reasons we light our streets, walkways, building entrances, parking lots and other areas. The problem comes when we choose, for whatever reason, to do the lighting with a poor choice of lighting fixture or installation. Too much of our night-time lighting worldwide is of poor quality, of poor design, or with no design at all. As such, it then compromises our goals, even to the extent of lowering visibility. Real safety is less, and we ruin the night-time ambience as well. In addition, since we are wasting a good deal of light, we waste expensive energy.

Quality lighting has none of these problems. It exists in many locations, and all good lighting designers and engineers (and most other people when they are aware of the issues) know the difference between good and bad lighting. 
While quality lighting might sometimes be more expensive than poor lighting, it is worth any such difference by the increased visibility, safety, security, and energy savings. It usually has a rapid payoff period. Good lighting has great value. There is no excuse for poor lighting. With good lighting, we all win.

\section{Why is Astronomy Interested in Night-Time Lighting?}

Think of the adjective "astronomical". What does it mean? There are two definitions: "having to do with astronomy", and "mind-boggling" (overwhelming the mind). We see it used in the media all the time in the latter context. We astronomers deal with mind-boggling things!

Astronomy is the Science of Extremes. Who can wrap their minds around distances of 15 billion light years, with light travelling at 186,000 miles a second. Eight minutes from the Sun, 5 years from the nearest other star. Our Galaxy, the Milky Way, is 100,000 light years in diameter; it is 2 million light years to the nearest galaxy, and over 15 billion light years to the farthest galaxies and quasars that we can observe. And we deal with the astronomically small too: atoms and nuclei. But not much in between. The same holds for temperatures: the highest in the Universe in the centres of some stars, and the lowest, in interstellar space. So it is with densities: some material in stars where an amount the size of my finger tip weighs more than all the people on Earth, and interstellar space where there are only a few atoms per cubic centimeter.

Black holes, quasars, cosmic rays, pulsars, even automobiles and computers get named after these things. Astronomy is an extremely interesting field. The public loves it. Do you know that there are more than 1000 times the number of amateur astronomers as there are professionals? How many amateur physicists or chemists do you know? Or amateur lighting engineers? There is something about astronomy in the press or on television almost every day. The media eats it up. Astronomy conferences have large press rooms and much coverage. How much coverage does street lighting get, unless there is a collision with a utility pole? Astronomy is a small field, but one with high visibility.

In addition to being a science and a frontier technology, astronomy is in many ways also a philosophy and an art, full of beauty and philosophical thinking. Where did we come from? What does the future hold? What does it all mean? These questions have been in front of humankind for millennia. And they always will be. It is fundamental and exciting stuff, truly mind-boggling. And think also of the potential for intelligent life elsewhere.

There are many beautiful images of things "out there". They regularly show up in the press and in magazines and on television. The Hubble Space Telescope as well as telescopes on the ground such as the VLT and the Keck telescopes have produced many stunning pictures, as well as fundamental research. Even small telescopes, even amateurs' telescopes, can do wonderful things. Essentially no. one is so blasé as not to be positively affected by these images and by many of the things going on in astronomy.

One of these mind-boggling things is how faint we work. On a human hand in a typical room there are about $1,000,000,000,000,000,000$ photons falling every second. With our telescopes for the faintest objects, we count photons one at a time and are almost always photon-limited in our studies. It is truly 
a frontier field of research. We are also at the cutting edge of technology, of course, for sensitive detectors and image processing. Much of this technology has applications to everyday life, such as in television and photometric applications.

So in relation to outdoor lighting, Astronomy is really the "Canary in the Mine". We notice the adverse impacts of bad lighting well before almost anyone else, and are strongly affected by such lighting. Two things in particular impact us: urban sky glow and local glaring sources. The latter is a major item for amateurs and the former for both amateurs and professionals. I will discuss both of these in some depth in the following sections of the paper.

Do dark skies have value? I think so. I think almost everyone else does too. Can we afford to lose our view of the stars and of the Universe? Can we toss away this heritage to our children and their children? No we cannot. It is sad to think that the only place most people can see a dark sky today is in a planetarium. The real thing is much better. Light pollution is definitely an environmental issue. Where are the environmentalists? Most have lost sight of the fact that the "day" is more than 12 hours long, that night is a key part of the environment.

In addition, over millenia humans have developed with a day-night cycle. By turning the night into day, we have added a psycho-social stressor to our system. Think about it. Do we need the break of the night? The contemplation of the stars and the Universe that our ancestors had? Too many of us have lost touch with nature, seeing it only on television or in the movies. There are things worth preserving and the dark sky is one of them.

The nice thing is that dark skies and quality lighting are compatible. The sad thing is that there is far too much bad lighting everywhere.

\section{Why the Problem?}

Simply put, there is a lot of bad night-time lighting. Far too much of it, everywhere. And such bad lighting is growing rapidly almost everywhere, much faster than the population.

What is bad lighting? We can define it by the following characteristics:

(i) Glare. Glare never helps visibility, yet is common in most outdoor lighting. Glare is never good. We should never tolerate it. It is not necessary. It can be avoided with good lighting design, in any installation.

(ii) Obtrusive lighting, or light trespass. This is our neighbor's light bothering us, or the local automobile dealer who has bad lighting, or the local sports complex with bad flood-lighting. There is far too much light trespass, obtrusive lighting. This lighting can even be offensive.

(iii) Clutter and confusion. This is light that does not add to the night-time ambience. It is a fact that too much of our night lighting actually helps to ruin the night-time environment. Many of us look forward to the time when we can bring back the beauty of the night that existed for many of our ancestors. 
(iv) Wasted light and urban sky glow. There is far too much up-going light, totally unused light, the major cause of our urban sky glow. In addition, there is the myth of "The More the Better". More light is not always better, no more than more salt is, nor more noise, nor more of almost anything. Certainly there are many locations with inadequate light, but there are also many with too much. The issue of transient adaptation (switching lights on only when they are actually needed, for example using motion sensors) is an important one and we must consider such possibilities in our lighting installations.

(v) Energy waste. Lots of energy (and money) is lost by all this wasted light and by inefficient lamps and fixtures. Billions of dollars, literally an astronomical amount, are wasted lighting up the sky and blinding us with glare.

\section{Why is There so Much Bad Lighting?}

The basic reason for so much bad lighting is that there is little awareness of the problems. Even though night lighting is all around us, most people actually see little of it. The bad stuff has crept up on us, little by little, with little notice, just like a cancer can creep up on us. Once we have begun to notice the problem, it is hard to do anything about it.

Then there is the additional problem of apathy, or perhaps we should call it inertia. Too many people have the attitude that poor lighting is there now and we can't do anything about it, or that it is too hard to change existing standards, or there is not time to be involved in the issues, or many other reasons. None of these reasons is good enough. The International Commission on Illumination (CIE) has addressed these issues, as have many national lighting organizations and their standard practices and recommendations are changing. Good lighting has great value and we must recognize it and market it. We can and should get rid of the old bad stuff and we should use only good lighting for all new installations. It is worth the difference in initial cost, if any. The long-term costs of new quality lighting are always lower than for the bad lighting.

While many push hard for more and brighter lighting as the key to solving the crime and security problem in our cities, we must note that all efforts in this direction so far have failed to solve the problem. In fact, the more we add light, the more crime seems to increase. The correlation between the increase in lighting and the increase in crime in most locales is excellent! But still we add more lighting in an attempt to do better. Maybe we are just adding more bad lighting, not helping. Good lighting can help, I am sure, but there is too little of it. All new recommendations from lighting organizations emphasize good lighting, not more lighting. Astronomers do not argue for no light, but for good lighting. 


\section{What can be Done?}

It is impossible to go into the many details here. Let me just review the solutions. They all go a long way to minimize light pollution and preserve dark skies without compromising in any way night-time safety, security, or utility.

1. Use night lighting only when necessary. Turn off lights when they are not needed. Timers can be very effective. Use the correct amount of light for the need, not overkill.

2. Direct the light downward, where it is needed. The use and effective placement of well-designed fixtures achieves excellent lighting control. Whenever possible, retrofit present poor fixtures. In all cases, the goal is to use fixtures that control the light well and minimize glare, light trespass, light pollution and energy usage.

3. Use energy efficient lighting and consider the use of low-pressure sodium (LPS) light sources, especially in the vicinity of major observatories. This is the best possible light source to minimize adverse sky glow effects on professional astronomy. LPS is especially good for street lighting, parking lot lighting, security lighting and any application where colour rendition is not critical. With creative design, it has even been used to illuminate new car dealerships.

4. Establish outdoor lighting ordinances that promote the use of quality lighting. Such controls do not compromise safety and utility. Lighting ordinances have been enacted by many communities worldwide and in several of the states in the USA, all designed to enforce the use of effective nighttime lighting and good design standards.

All of these solutions to the problem say: "Do the best possible lighting design for the task. Always consider and minimize all the relevant adverse factors, such as glare, light trespass and urban sky glow." All the solutions needed for protecting astronomy have positive side benefits of maximizing the quality of the lighting, improving visibility and saving energy. We all win!

\section{Conclusions}

There is a problem.

The problem is for all of us, not just astronomers.

The problem is still getting worse almost everywhere.

Why do we tolerate it?

Lack of awareness. Apathy. Too many laws.

So bad now that nothing can be done.

No time to work on it. 
None of these reasons is good enough!

None will help us solve the problem or get solutions.

We must do something now!

We know that working solutions exist.

Awareness and education are the keys to getting action.

Quality lighting is the key!

It is an issue in which everyone can win.

Why shouldn't we do it?

The Goals:

- Dark skies.

- Quality lighting, with better visibility at night, hence better safety and security.

- Better night-time ambience.

- Considerable energy savings.

\section{Let's do it!}

\section{References}

Astronomical Society of the Pacific, The Universe in the Classroom, No.44Fourth Quarter, ASP, 1998

CIE 1997 "Guidelines for minimzing sky glow", CIE Technical Report 126

Crawford, D. L. (Ed.) 1991, IAU Colloquium No. 112, Light Pollution, Radio Interference, and Space Debris, Astronomical Society of the Pacific Conference Series, Vol. 17

Isobe, S. and Hirayama, T. (Eds.) 1998, Preserving the Astronomical Windows Astronomical Society of the Pacific Conference Series, Volume 139.

See the IDA Web Page http://www.darksky.org/ ida for much additional information and for links to other resources. 


\title{
Controlling Light Pollution in Chile: A Status Report
}

\author{
Malcolm G. Smith \\ AURA/Cerro Tololo Interamerican Observatory, La Serena, Chile
}

\begin{abstract}
A basic-level summary is provided of work since late 1993 to control light pollution in Chile. The purpose of this article is to stimulate such work inside Chile and to promote good lighting in developing countries in general. Chile is selected as the case study because of its critical importance to optical and radio astronomy, and the related economic and cultural benefits for Chile and the world. Examples are presented in some detail in order to illustrate adjustments that have been made to accommodate local scientific, cultural and economic realities and to show that it is necessary to anticipate the issues involved in controlling light pollution several decades before it would otherwise become a problem. It is hoped that international organizations such as the IAU, the IDA and the CIE can soon promote programmes in Chile that can serve as pilot programmes for other parts of the developing world.
\end{abstract}

\section{Introduction}

I was invited by the organizers of this Symposium to give a presentation on "The Importance of Ground-Based Optical Astronomy" suitable for lighting engineers and astronomers. The opening remarks by speaker after speaker emphasized the key scientific, economic and cultural aspects of ancient and modern astronomy. Being unwilling to dissociate ground-based from space-based astronomy or optical- from radio- and millimeter-wave astronomy, my talk emphasized the importance of ground-based astronomy within this wider, integrated context.

The scientific importance of astrophysics as a laboratory of extreme conditions, e.g. in solar flares, planetary nebulae, supernovae, the nuclei of galaxies, gamma-ray bursters, black holes and the big bang is known to all physicists and astronomers.

Its cultural importance in terms of understanding our origins and eventual fate is well known to all. Well-written and authoritative treatments of these matters at the level of a general reader can be found in books on cosmology such as those by Weinberg (1993), Hawking (1988) and Rees (1997).

The economic and cultural importance, over the centuries, of knowledge of the seasons, navigation, gravity, nuclear energy and so forth is obvious. Practical spin-offs from modern astronomical technology include such diverse areas as advances in medical x-ray technology, breast-cancer diagnosis, monitoring of the structural integrity of oil rigs and the avoidance of waste light energy from cities. 
However, following the various discussions at the Symposium, it became clear that a volume like this would be more useful if it contained some discussion of the issues involved in preserving the astronomical sky - as they arise today in Chile. This is because Chile is at the centre of the greatest construction programme ever undertaken in ground-based astronomy, so the scientific, economic and cultural importance of its astronomical sky is obvious and increasing. Chile also provides examples of issues associated with controlling light pollution in a developing country.

The preservation of the astronomical skies over Chile is a concern for everyone - and an appropriate issue, therefore, for the IAU and other international bodies (such as the UN, UNESCO, the Commission Internationale d'Eclairage and the International Dark-Sky Association), as well as for Chile's own national and regional authorities.

Astronomy has, over the years, found it necessary to run further and further away from industrialized areas in order to be able to work under skies unpolluted by man-made interference. Other, more qualified speakers have addressed the issue of radio interference; with the advent of the Atacama Large Millimeter Array (ALMA), control of such interference is obviously vital to international astronomy and to the economics of that region of Chile. This basic-level article will be focussed on optical interference - primarily the increase over natural sky background produced by light from surrounding communities and industries.

\section{Light Pollution in Chile - Scientific Aspects}

Seven telescopes larger than $6 \mathrm{~m}$ in diameter are currently being brought into regular scientific use in northern Chile or are nearing their final stages of construction and commissioning. The four 8-m elements of the European Southern Observatory's Very Large Telescope (VLT) on Cerro Paranal, the Carnegie Institute of Washington's twin 6.5-m Magellan telescopes on Cerro Las Campanas and the international 8-m Gemini South telescope on Cerro Pachón will all depend for their success on the skies over northern Chile being preserved in their current pristine condition. New generations of large, wide-field, survey telescopes such as the VLT $2.65 \mathrm{~m}$ Survey Telescope (VST), the British 4-m VISTA telescope and the US 8-m Dark-Matter Telescope are being built or proposed specifically to take advantage of the darkest available skies.

Examples of the kinds of science that need such dark skies cover the full range of size and distance in the universe - from the detection of large-scale structure by means of weak gravitational lensing of faint galaxies to the early location and study of near-earth objects (asteriods and comets).

To set a context for the goal of the efforts to preserve these dark astronomical skies, the natural sky background at new moon near the zenith at high ecliptic and galactic latitudes varies by a factor of about 1.7 , i.e. by about $0.6 \mathrm{mag},(21.3<V<21.9)$ per square arcsecond over the course of the 11year solar activity cycle (Krisciunas 1997). This minimum sky brightness is "about 10 million times dimmer than the daylight sky (but easily visible to the dark-adapted eye)" (Benn and Ellison 1999).

Faint galaxies will be observed with telescopes in Chile down to surface brightness levels at or even below $29 \mathrm{mag}$ per square arcsecond, i.e. $\sim 700$ 
times fainter than the natural solar-minimum background at V. Our goal must be to ensure that artificial light does not increase the natural sky background by more than about 0.1 magnitudes per square arcsec at these levels of surface brightness, at least in broad passbands (Smith 1979; Krisciunas 1997).

AURA's observatory is the closest major observatory in Chile to city lights (see section 5). It is therefore a natural point from which to lead an effort to study and control light pollution, well before it can become a serious problem at Cerro Pachón and Cerro Tololo.

The measured sky brightness at the zenith at Cerro Tololo is $<0.08 \mathrm{mag}$ per square arcsecond brighter than the natural value, i.e. it is not yet possible to measure any artificial light pollution at zenith distances $<45$ degrees through broad-band filters (see, for example, Walker and Smith 1999). The sky overhead at Cerro Tololo and Cerro Pachón is still very dark.

Nevertheless, the ability to detect the sodium lights of La Serena spectroscopically by looking low in the sky in the direction of that city is an early warning that action that started in earnest in late 1993 has to continue, especially as such action has implications for astronomy throughout Chile. It may also be possible to detect such radiation in certain directions low over the horizon from other major observatories in Chile. This should be attempted now at all of the major optical observatories (both in Chile and elsewhere) and monitored regularly. An excellent example of such work in La Palma has been described recently by Benn and Ellison (1999).

As discussed in section 5.1 , it has already taken nearly 6 years to secure a set of government lighting regulations that can now be taken to local municipalities for communication, education, development of municipal ordinances and eventual enforcement. It is necessary to anticipate the issues involved in controlling light pollution several decades before it would otherwise become a problem.

\section{Light Pollution in Chile - Economic Aspects}

The current boom in construction of international astronomical facilities in the north of Chile involves investments already surpassing a billion dollars. Specific plans exist which take this figure to well over US\$1.2 billion. The detailed budgets for the observatories involved are, unfortunately, not readily available. Nevertheless, from experience, one can make rough estimates for the level of investment made in the Chilean economy. These lead to averages of about US\$60M per annum entering the economy for construction and a steady rampup to about US\$40M of annual investment in operations.

The Chilean national economy thus receives about US\$100M in annual income from astronomy. Chilean astronomers are, in addition, awarded $10 \%$ of the observing time on telescopes in Chile. In return, the Chilean government provides a variety of privileges and tax breaks to the international astronomical community, and is generally supportive of astronomical development and the associated benefits arising from this foreign investment.

The AURA Observatory in Chile (Gemini South, SOAR and the telescopes on Cerro Tololo) provides a measure of the economic stimulus that occurs at a regional level. AURA has brought approximately US $\$ 150 \mathrm{M}$ in construction projects to Region IV of Chile and currently spends about US $\$ 4 \mathrm{M}$ dollars each 
year in Chile on operations. From 2002 onwards, with the arrival of Gemini South and SOAR, AURA will be spending about US\$7M per annum in Chile. AURA has also stimulated local entrepreneurial activity in astronomical tourism, education and research as described in more detail in section 5 .

\subsection{DMSP Data as a Monitor of Energy Waste}

We have seen that Chile has a strong economic interest in preserving the astronomical sky. Further beneficial economic and environmental impact arises from the potential for more rational use of energy in exterior lighting. As we have heard elsewhere at this conference, the United States shines well over US $\$ 1$ billion of light annually into the heavens, while each year Japan inadvertently sends about US $\$ 200 \mathrm{M}$ dollars worth of light into the sky (Isobe and Hamamura 2000).

It will be interesting over the next few years to extend the use by Elvidge and collaborators of unsaturated Defense Meteorological Satellite Program (DMSP) data from the National Geographic Society's databank (Luginbuhl, personal communication; see also Cinzano et al.(2001), and Isobe, Hamamura and Elvidge (2001)). Carefully selected subsets of these data should permit measurement and monitoring of the upwardly-directed light from Chilean cities and towns, including those nearest the major observatories.

One may be able to stimulate a positive reaction from authorities in developing countries by providing information on the luminous flux, expressed in terms of wasted money, from their major cities; this information could be accompanied by reasonably simple guidelines, in their own language, about what can be done to reduce the waste. Isobe and Hamamura (2000) provide an example of this idea applied to an industrialized nation. Santiago de Chile would be a good initial test case, given the existing positive attitude of authorities at the national level and a recent awareness of the need to conserve electrical energy (brought on by a drought in the southern lake region which provides most of the country's hydroelectric power).

Early DMSP data were taken at high gain. The data from large cities were saturated. Unsaturated data from the DMSP may, however, be available from the outset for smaller, isolated towns - such as those near major observatories. It will be a challenge to see if sufficient precision and accuracy in calibration can be obtained to monitor changes in the upward flux as remedial lighting programmes come into effect.

\subsection{Astronomical Tourism}

Chile is beginning to realise the potential for eco-tourism based on its dark skies. The Servicio Nacional de Turismo (SERNATUR) has recently produced a 57page full-colour booklet on astronomical tourism in the province of Elqui (where AURA has its observatory). In combination with the municipal observatory described in section 4.3 , they are generating sufficient funds from publicizing the dark skies in the region to support a growing tourist industry. 


\section{Light Pollution in Chile - Cultural Aspects}

Several speakers at the Symposium mentioned the ancient cultural links in many parts of the world between astronomy, different religions and practical pursuits such as agriculture and navigation. In addition to the cultural impact of the tourism mentioned above, astronomy provides humankind, on our tiny island planet, with a sense of perspective on our place in the universe.

\subsection{The Voyage of the Hokule'a}

At this time, the Polynesian Voyaging Society's canoe "Hokule'a" (Arcturus) is sailing to Rapa Nui, better known as Easter Island (the most westerly part of Chile). This journey is the culmination of a 25-year-long series of voyages by PVS's canoes across Polynesia; these voyages have led a cultural renaissance in the region. This latest voyage will close the Polynesian Triangle, formed by New Zealand, Hawai'i and Easter Island. The seas (away from the squid fleets - Sullivan 2001) offer dark skies to the navigators.

To quote from the PVS web page, "To get from Hawai'i to Rapa Nui, Hokule'a must travel.., 2820 nautical miles south (from 20 degrees $\mathrm{N}$ to 27 degrees S) and 2760 nautical miles east (from 155 degrees $W$ to 109 degrees). The first three destinations (Nukuhiva, Mangareva and Rapa Nui) lie upwind of the departure points, so the canoe will have to struggle to get east against the prevailing winds."

"The sail from Mangareva to Rapa Nui will be the most difficult, as Rapa Nui lies 1450 miles to the east of Magareva...On this leg, Hokule'a will be navigated without instruments by a team of Hawai'i's best navigators, headed by Nainoa Thompson. They will guide the canoe by celestial bodies (sun, moon, planets and stars), ocean swells...."

These bold navigators use astronomy in their endeavors and convey the wisdom of the more successful island peoples about their relationship to the environment. The Earth, too, is a tiny, isolated island; dark starlit skies help remind the whole of humanity of our fragile existence in one, much vaster, universe (Rees 1997).

\section{Details of Work on Light Pollution in Chile}

\subsection{Work at National and Regional Levels: Development of Regu- lations and Municipal Ordinances in Chile}

At about the time of IAU Symposium 196, Chile's President Eduardo Frei RuizTagle signed the "Norma Luminica" which gives the force of law to a set of lighting regulations covering Regions II, III and IV of (Northern) Chile. Cerro Paranal is in Region II. Cerro La Campanas is in Region III. Cerro La Silla, Cerro Tololo and Cerro Pachón are all in Region IV. This code is currently being translated into English and will be available on the world-wide web by the time this article is published (www.ctio.noao.edu/ctio.html).

The Chilean National Environment Commission, CONAMA, is leading a major programme of outreach and training related to the "Norma Luminica", in conjunction with the major international optical observatories in Chile. A 
series of light-pollution "events" will be held later this year, one in each of the three astronomically-critical regions.

Each event will include an outreach workshop organized by CONAMA, targeting the general public, local government officials and representatives of companies in the private (commercial) sector. The events will also include a technical training workshop, organized in close consultation with one of the observatories in the relevant region.

CONAMA is also organizing, in consultation with the international observatories, the production of 1,000 copies of an "Application manual" for the "Norma Luminica", along with a special web page with links from the CONAMA home page and links to various observatories and key websites on light pollution.

The first regional CONAMA office of technical support for the "norma" is to be set up in La Serena for a period of two years, with personnel financed by the international observatories in Chile. After 2 years, the value and performance of this office will be assessed. One of its early tasks will be to create a database on sources of light pollution in the three critical regions of Chile.

This set of national regulations provides a framework which allows us now to start serious work with municipalities in Chile, with the aim of mitigating and controlling light pollution. The President of the IVth Chapter of the Chilean Association of Municipalities (which includes those closest to AURA's Observatory) has recently and publicly expressed a wish to help in this effort in specific and significant ways.

\subsection{Work at Local Level: 1. Vicuña and its Municipal Observatory at Cerro Mamalluca}

Initial efforts five years ago to capture the attention and support of local municipalities did not go so well. The initial reaction was that changing exterior light fixtures would be too expensive, that tourism was flourishing as a result of the bright lights along the beaches and that there were concerns to ensure security at night. Although we used the correct reasoning and examples provided by the IDA, we did not make progress until an employee of the municipality of Vicuña contacted us (Sr. Eduardo Valenzuela, who is also an amateur astronomer). Vicuña is the closest town to Cerro Tololo and Cerro Pachón, with a central population of about 10,000 (excluding outlying regions) at line-of-sight distances of roughly $18 \mathrm{~km}$ and $23 \mathrm{~km}$ from Tololo and Pachón, respectively. As luck would have it, municipalities in Chile were being offered the chance to apply for a loan from the central government to replace the old, inefficient mercury fixtures with energy-efficient sodium fixtures. The two amateurs asked us to help the municipality design the fixtures in such a way as to minimize running costs (in particular) and minimize the impact on the astronomical research going on nearby! This was the breakthrough we were looking for.

We first studied the situation in the USA, with the help of the IDA materials and Dave Crawford's personal advice. We soon realized that what works in the USA may not satisfy Vicuña's requirements. At that time there was only one supplier of low-pressure sodium lamps in the country. Such fixtures were too expensive for most Chilean municipalities because of losses to vandalism. Highpressure lamps have a wider market and are therefore available in Chile at lower capital cost. 
We then made contact with Sr. Enrique Piraino, a lighting engineer from the Universidad Catolica de Valparaiso, who runs a laboratory used to measure and standardize Chilean lighting fixtures available on the Chilean market. Sr. Piraino provided consultant services to the Municipality of Vicuña (at AURA expense) in order to replace and reposition the town's street lights. His recommendations included replacing all the white-light globes which lit the town's main street, the "Avenida de las Delicias". The first step was to draw up an inventory of all existing street lights in the town (exisiting inventories were so inaccurate as to be useless). Powerful ( $400 \mathrm{~W})$ full-cutoff fixtures were used to illuminate the perimeter of the town square. $150 \mathrm{~W}$ fixtures were used for the main streets near the centre of town. Remaining streets were lit with $70 \mathrm{~W}$ fixtures, all high-pressure sodium.

A rough calculation shows that the lighting change and redesign saves Vicuña nearly half of its municipal lighting bill. 1534 light fixtures were changed or modified. Illumination increased from $7.8 \mathrm{M}$ lumens to $8.9 \mathrm{M}$ lumens. Estimated power consumption dropped from $210 \mathrm{~kW}$ to $110 \mathrm{~kW}$, with consequent large savings. Estimated power consumed in illuminating the sky dropped from $24 \mathrm{~kW}$ to just over $1 \mathrm{~kW}$. The capital cost of the modernization has already been paid off from these savings and the municipality is delighted.

In recognition of Vicuña's seminal co-operation, the US National Science Foundation, at AURA's recommendation, donated a $30-\mathrm{cm}$ commercially-made telescope to Vicuña as the centrepiece of a municipal observatory. The town found the land, installed a $4 \mathrm{~km}$-long road, provided power, water and a building (which did not get funding from the Chilean science foundation, CONICYT but did get a large grant from the Chilean National Fund for Art and Culture in addition to local, commercial sponsorship). Cerro Tololo provided an obsolete dome that was in good condition.

AURA staff provided the first course of lectures and practical workshops to members of the local community. Graduates of this first course then taught the second course in the series, so that the town now has people qualified to help visitors in various aspects of the observatory.

The new Cerro Mamalluca Municipal Observatory is now making sufficient money from tourism ("come to the darkest, clearest skies in the world") and conferences to be almost self sufficient and, with the help of government grants, will shortly be buying several new telescopes of its own. Phase I, the telescope, building and dome, is in regular operation, every clear night. Phase II, containing a restaurant and a platform for amateur telescopes, is nearly complete. A small-telescope educational and dark-sky research facility is at the proposal stage for Phase III; funds for this third phase are expected to be awarded next year.

Vicunã has worked closely with AURA to become a demonstration community, allowing us to present a way to develop a mutually-beneficial communitywide business approach to the provision of good lighting. Once the cost of international broadband links drops sufficiently, we hope to extend this work to include connection between schools in Chile and those in other countries (initially from the Gemini and SOAR consortium partnerships). Among other plans being discussed are the production of a CD or video, in Spanish, on the subject 
of light pollution, using illustrations of lighting in the region. This work is likely to be done in close collaboration with M. Metaxas (see paper at this conference).

\subsection{Work at Local Level: 2. Other Lighting Projects near the AURA Observatory in Chile}

The nearest town to the AURA Observatory in Chile is Andacollo, at a distance of only $28 \mathrm{~km}$. from Cerro Tololo and $33.5 \mathrm{~km}$ from Cerro Pachón. Although it has only $\sim 12,000$ inhabitants, two international open-pit mines have opened there in the last five years. Fortunately, the lack of vandalism in the controlled area of a mine allows the mine to make economic use of the most energy efficient low-pressure sodium lighting. The mine needs powerful lighting for safe operation; therefore the potential for significant light pollution from such a nearby source, particularly during the early phases of the project, was serious. Fortunately the mine was looking for opportunities to improve its rather tarnished environmental reputation and worked closely with us to direct its lights away from the line of sight to the observatory. We even identified troublesome individual fixtures for them on the telephone, as they kindly switched their lights off and on for us, one by one.

The nearest cities form the coastal conurbation of La Serena and Coquimbo, at a distance of $\sim 49 \mathrm{~km}$ from Cerro Tololo (and $\sim 58 \mathrm{~km}$ from Cerro Pachón) and containing about 292,000 inhabitants. Chile's recent economic boom has increased the estimated output of light per inhabitant to levels similar to those in industrialised nations. Fortunately, both La Serena and Coquimbo are largely hidden from direct view from Cerro Tololo and Cerro Pachón by a range of mountains paralleling the coastline just a few kilometers from the shore. The arrival of bright lights, especially those along the beach drive (the "Avenida del Mar") has provided an undeniable stimulus to the economics of local tourism.

AURA has had to proceed very carefully to search for ways in which lighting can be used to mutual benefit (see, e.g., Mendez and Boccas 1999). This proved difficult at first, but efforts to set up a sister-city exchange between the county of Hawai'i and La Serena have allowed us to demonstrate that massive tourism and good lighting can coexist elsewhere and that we should be able to work together in Chile to achieve this. Economic arguments have so far been much more persuasive than cultural and scientific arguments, although even these are now gaining force as the tourist potential of dark skies becomes better understood.

Coquimbo is currently engaged in construction of a US\$6M "Cruz del Tercer Milenio" on a prominent hill overlooking the harbor. This is directly visible from AURA's observatory. The recent publication of the national lighting regulations has undoubtedly helped us in work with the municipality to design downwardlooking light fixtures for this 80 -meter-high religious structure.

\section{Conclusions - Monitoring the Sky Background}

Lighting engineers and others at this symposium with the qualifications and the will to help have repeatedly expressed their frustration that professional astronomers have published so little information on the night sky background that engineers could use to check cause and effect in light pollution. AURA will 
again be taking the lead on the professional side in trying to contribute to an improvement in this situation.

Tololo and Pachón can be seen as a "canary in the mine" for modern astronomy in the southern hemisphere, just as astronomy itself acts in a similar way to provide early warning to the rest of humankind. Even a worst-case scenario with aggressive population growth and no lighting control measures would predict an increase of $\sim 0.1 \mathrm{mag}$ above the natural solar-minimum background over AURA's observatory (21.9V mag per square arcsecond, or 58 nanolamberts, at the zenith) by 2020 (Walker and Smith 1999). Dave Crawford and the IDA have shown, in Tucson, what can be done to contain the effects of such population growth. A more realistic model shows that, even by 2030 , light pollution at the zenith will still be only $\sim 0.08 \mathrm{mag}$ (to be compared with the $0.6 \mathrm{mag}$ change in natural background during the solar cycle). This, however, does assume some success at light pollution control. We are working hard on such controls in Chile; the canary is not going to die readily.

Acknowledgments. The early efforts of the late Dr. Art Hoag in Tucson, Victor Blanco in Chile and the sustained efforts everywhere of Dave Crawford and the International Dark-Sky Association provided a vital foundation for this work. Sr. Edgardo Boeninger K. (then Minister/Secretary General to the Chilean President) explained to me in late 1993 the need to contact the Comision Nacional del Medio Ambiente (CONAMA), the newly-formed national environment agency. This national-level approach contrasted with the exclusively localand regional-level emphasis that has proved quite effective in many parts of the USA (Davis 2001).

Sr. Rodrigo Egaña, the National Director of CONAMA, has proved a staunch and constant supporter of light-pollution control (following a visit to Cerro Tololo at night). Sr. Pedro Sanhueza, the director of COREMA (the regional version of CONAMA for Region IV of Chile) and ex officio member of the CONAMA, took repeated risks in coming out publically at regional and national level in support of light pollution control. Sra. Cecilia Prats, regional director of SERNATUR, the Chilean national tourism agency, was among the first to press effectively for marketing the dark skies in the region as a non-seasonal tourist attraction. Sr. Enrique Piraino provided technical assessment appropriate to the realities confronting lighting engineers in Chile.

The Cerro Tololo Light Pollution Control Committee was chaired most effectively for much of the last five years by Dr. Mark Phillips. He and his colleagues, Sres. Ricardo Schmidt, Mario Hamuy, Oscar Saa, M. Maxime Boccas and Dr. Rene Mendez (the current committee chair), must take most of the credit for what has been achieved from the astronomers' side in Chile.

This effort would have hardly started without the vision and persistence of the amateur astronomers and municipal authorities in Vicuna, Chile.

\section{References}

Benn, C. R. and Ellison, S. L. 1999, submitted .

Cinzano, P., Falchi, F., Elvidge, C. D. and Baugh, K. E. 2001, this conference.

Davis, D. R. 2001, this conference. 
Hawking, S.W. 1988, A Brief History of Time, Bantam (Random House) New York.

Isobe, S., Hamamura, S. and Elvidge, C. 2001, this conference.

Isobe, S. and Hamamura, S. 2000, Memorie della Società Astronomica Italiana, 71, No. 1, 131.

Krisciunas, K. 1997, P.A.S.P., 109, 1181.

Mendez, R. and Boccas, M. 1999, NOAO Newsletter No. 59 (Sept.), 23.

Rees, M. 1997, Before the Beginning: Our Universe and Others, Perseus Books.

Sullivan, W. 2001, this conference.

Walker, A and Smith, C. 1999, NOAO Newsletter No. 59 (Sept.), 21.

Weinberg, S. 1993, The First Three Minutes, Basic Books.

CTIO www.ctio.noao.edu (contains links to the NOAO Newsletter, Gemini, SOAR, VLT, Magellan and the Vicuña municipal observatory).

IDA www.darksky.org

PVS leahi.kcc.hawaii.edu/org/pvs 